\title{
The ability of streptomycin-loaded chitosan- coated magnetic nanocomposites to possess antimicrobial and antituberculosis activities
}

This article was published in the following Dove Press journal:

International Journal of Nanomedicine

30 April 2015

Number of times this article has been viewed

\author{
Mohamed Ezzat El Zowalaty ${ }^{1,2}$ \\ Samer Hassan Hussein Al Ali $\mathrm{i}^{3,4}$ \\ Mohamed I Husseiny ${ }^{2,5}$ \\ Benjamin M Geilich 6,7 \\ Thomas J Webster ${ }^{7,8}$ \\ Mohd Zobir Hussein ${ }^{9}$ \\ 'Laboratory of Vaccines and \\ Immunotherapeutics, Institute of \\ Bioscience, Universiti Putra Malaysia, \\ Selangor Darul Ehsan, Malaysia; \\ ${ }^{2}$ Department of Microbiology and \\ Immunology, Faculty of Pharmacy, \\ Zagazig University, Zagazig, Egypt; \\ ${ }^{3}$ Laboratory of Molecular Biomedicine, \\ Universiti Putra Malaysia, Selangor \\ Darul Ehsan, Malaysia; ${ }^{4}$ Faculty of \\ Pharmacy, Isra University, Amman, \\ Jordan; ${ }^{5}$ Beckman Research Institute \\ of City of Hope, Duarte, CA, USA; \\ ${ }^{6}$ Department of Bioengineering, \\ ${ }^{7}$ Department of Chemical Engineering, \\ Northeastern University, Boston, \\ MA, USA $;{ }^{8}$ Center of Excellence for \\ Advanced Materials Research, King \\ Abdulaziz University, Jeddah, Kingdom \\ of Saudi Arabia; ${ }^{9}$ Materials Synthesis \\ and Characterization Laboratory, \\ Institute of Advanced Technology; \\ Universiti Putra Malaysia, Selangor \\ Darul Ehsan, Malaysia
}

Correspondence: Mohamed Ezzat El Zowalaty

Laboratory of Vaccines and

Immunotherapeutics, Institute of

Bioscience, Universiti Putra Malaysia,

Serdang, 43400 UPM, Selangor Darul

Ehsan, Malaysia

Email elzow00I@gmail.com

Mohd Zobir Hussein

Materials Synthesis and Characterization Laboratory, Institute of Advanced

Technology, Universiti Putra Malaysia,

Serdang, 43400 UPM, Selangor, Malaysia

Tel +6038946680 l

Fax +60389435380

Email mzobir@upm.edu.my
Abstract: Magnetic nanoparticles (MNPs) were synthesized by the coprecipitation of $\mathrm{Fe}^{2+}$ and $\mathrm{Fe}^{3+}$ iron salts in alkali media. MNPs were coated by chitosan (CS) to produce CS-MNPs. Streptomycin (Strep) was loaded onto the surface of CS-MNPs to form a Strep-CS-MNP nanocomposite. MNPs, CS-MNPs, and the nanocomposites were subsequently characterized using X-ray diffraction and were evaluated for their antibacterial activity. The antimicrobial activity of the as-synthesized nanoparticles was evaluated using different Gram-positive and Gram-negative bacteria, as well as Mycobacterium tuberculosis. For the first time, it was found that the nanoparticles showed antimicrobial activities against the tested microorganisms (albeit with a more pronounced effect against Gram-negative than Gram-positive bacteria), and thus, should be further studied as a novel nano-antibiotic for numerous antimicrobial and antituberculosis applications. Moreover, since these nanoparticle bacteria fighters are magnetic, one can easily envision magnetic field direction of these nanoparticles to fight unwanted microorganism presence on demand. Due to the ability of magnetic nanoparticles to increase the sensitivity of imaging modalities (such as magnetic resonance imaging), these novel nanoparticles can also be used to diagnose the presence of such microorganisms. In summary, although requiring further investigation, this study introduces for the first time a new type of magnetic nanoparticle with microorganism theranostic properties as a potential tool to both diagnose and treat diverse microbial and tuberculosis infections.

Keywords: iron oxide nanoparticles, chitosan, streptomycin, Mycobacterium tuberculosis, antimicrobial activity

\section{Introduction}

Nanomaterials are compounds of interest in numerous fields, including physics and chemistry, because the reduced dimension of a solid material (such as a particle, grain, tube, etc) leads to novel, unexpected physical and chemical properties that differ from that of the corresponding bulk material. ${ }^{1}$ The foundation of these unexpected properties is due to the large surface-to-volume ratio of these nanoparticles, compared to their micron equivalents. ${ }^{2}$ Iron oxide nanoparticles (for example, magnetite or $\mathrm{Fe}_{3} \mathrm{O}_{4}$ ) are an example of an ultra-small nanomaterial, which are beginning to revolutionize science. ${ }^{3}$ In addition, iron oxide nanoparticles are promising candidates for drug delivery, ${ }^{4}$ contrast enhancers in magnetic resonance imaging (MRI) medical diagnostics, ${ }^{5}$ and are promising antimicrobial agents to combat bacteria resistant to conventional antibiotics. ${ }^{6}$

Infections caused by antibiotic resistant microorganisms are increasing globally, reaching epidemic proportions. ${ }^{7}$ Antimicrobial resistance to various classes of antibiotics is on the rise, with the emergence of multiple drug resistance among different microorganisms raising great concern in clinical settings. ${ }^{8,9}$ The escalating rate of microbial resistance to several different classes of antimicrobial agents raises significant global 
concerns for health care and public health practitioners. ${ }^{10}$ There are looming bacterial outbreaks due to pandrug-resistant pathogens that may cause fatal infections among humans and animals. Nano-antibiotics are novel drug candidates of promise to combat both microbial infections and the increased bacterial resistance to antibiotics. Nano-antibiotics provide efficient anti-infective agents for biomedical and therapeutic applications. Nano-antibiotics are considered a potential treatment option to control infectious diseases and to reduce the overwhelming microbial resistance prevalent nowadays. ${ }^{11,12}$ During the past decades, new infections have emerged, and older infections previously thought to be controlled have re-emerged; hence, infectious diseases continue to be one of the greatest health care challenges worldwide. Numbers of multidrug-resistant bacteria are rising steadily, and the current pool of antimicrobial agents is becoming less efficient in combating resistant microbes. ${ }^{13}$

Previously, streptomycin (Strep)-chitosan (CS) magnetic nanocomposites were developed by this research group, and they were characterized by determining preliminary antibacterial activity. ${ }^{14}$ However, in the present study, we expand on such results and show for the first time potential anti-tuberculosis (anti-TB) activity. Since the placement of magnetic nanoparticles (MNPs) can be controlled through a magnetic field, and their presence identified via MRI, such an approach offers incredible promise for the development of unique theranostic tools (that is, an approach that can allow simultaneous diagnosis and treatment of microorganisms) for microbial infections.

\section{Materials and methods}

\section{Reagents and chemicals}

All chemicals used were of analytical reagent grade and were used without further purification. CS (low molecular weight, deacetylation 75\%-85\%) was purchased from Sigma-Aldrich (Saint Louis, MO, USA). Ferric chloride $\left(\mathrm{FeCl}_{3} \cdot 6 \mathrm{H}_{2} \mathrm{O}\right.$ ) with $99 \%$ purity and ferrous chloride $\left(\mathrm{FeCl}_{2} \cdot 4 \mathrm{H}_{2} \mathrm{O}\right)(>99 \%$ purity) were obtained from Merck KGaA (Darmstadt, Germany), sodium hydroxide (28\% purity), and Strep sulfate were obtained from Sigma-Aldrich. An acetic acid solution (99.8\% purity) was purchased from Hamburg Industries Inc (Hamburg, Germany). All aqueous solutions were prepared using deionized water.

\section{Synthesis of bare, CS-coated MNPs and Strep-loaded CS-coated MNPs}

The syntheses of bare, CS-coated MNPs (CS-MNPs) and streptomycin-loaded, CS-coated MNPs (Strep-CS-MNPs) were based on a previously reported method. ${ }^{14,15}$ The method involves co-precipitation of $\mathrm{Fe}^{2+}$ and $\mathrm{Fe}^{3+}$ iron salts in aqueous solution by adding sodium hydroxide (2 M). In brief, $2.43 \mathrm{~g}$ of ferric ion and $0.89 \mathrm{~g}$ of ferrous ion were dissolved in deionized water. After that, $20 \mathrm{~mL}$ of sodium hydroxide was rapidly added to precipitate iron oxide nanoparticles, and the color of the solution mixture turned from light orange to black. The $\mathrm{pH}$ was kept to not less than 10 at room temperature. The solution was sonicated for 60 minutes at room temperature. The MNPs were filtered, washed three times with deionized water, and dried.

CS-MNPs were prepared as follows: $2.0 \mathrm{~g}$ of CS was dissolved in a $1 \%$ acetic acid solution. The CS solution and MNPs were mixed together in a flask, and the mixture was stirred for 18 hours. CS-MNPs were separated using a permanent magnet and dried at $70^{\circ} \mathrm{C}$ for 2 hours.

A Strep-CS-MNP nanocomposite was prepared as follows: a Strep solution $(0.58 \mathrm{~g})$ was mixed with aqueous dispersions of MNPs. The mixture of CS-MNPs in the Strep solution was magnetically stirred at room temperature for 18 hours to facilitate Strep uptake. The Strep-CS-MNP nanocomposite was separated using a permanent magnet.

\section{Characterization of Strep-CS-MNP nanocomposite}

The MNPs and Strep-CS-MNP nanocomposite were characterized using X-ray diffraction (XRD). XRD spectra were obtained in the range of $28^{\circ}-65^{\circ}$ using an XRD-6000 diffractometer (Shimadzu, Tokyo, Japan) with $\mathrm{CuK} \alpha$ radiation $(\lambda=1.5406 \AA)$ at $30 \mathrm{kV}$ and $30 \mathrm{~mA}$.

\section{Evaluation of antimycobacterial activity of Strep-CS-MNPs}

The drug susceptibility testing (DST) of Strep-CSMNPs using the Mycobacteria Growth Indicator Tube (MGIT'T) 960 against Mycobacterium tuberculosis (American Type Culture Collection (ATCC) ${ }^{\circledR} 25618^{\text {TM}}$; ATCC, Manassas, VA, USA) was used, and the minimum inhibitory concentration (MIC) of the nanocomposite

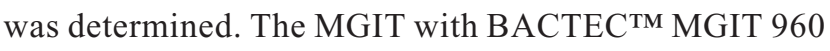
growth supplement for DST was used in the MGIT 960 instrument (BD, Franklin Lakes, NJ, USA), as described previously. ${ }^{16,17}$ All media and reagents were supplied by the manufacturer. The standard protocol for DST in MGIT 960 was strictly followed, as recommended for primary drugs. Culture suspensions for inoculation were 
well dispersed, with no large clumps, necessary to avoid false-resistant results.

After thorough mixing and homogenization of the culture suspensions, the tubes were allowed to rest for at least 15 minutes, and the supernatant was used to inoculate the drug-containing media and the control, according to the manufacturer's instructions for DST of first-line drugs. All inoculated drug-containing MGIT 960 tubes were placed in the DST set carrier and entered into the MGIT 960 instrument as "unknown drugs" using the DST entry feature. For the DST set containing "unknown drugs", the instrument flagged the DST set "complete" when the growth control reached a growth unit (GU) value of 400 . At that point, the GU values of drug-containing tubes were retrieved from the instrument by printing out a DST set report, and results were interpreted manually. If the GU of the drug-containing tube was more than 100 when the GU of the growth control was 400, the results were defined as resistant. If the GU values of the drugcontaining tubes were equal to or less than 100, the results were considered susceptible. Experiments were repeated with various concentrations of the Strep-CS-MNP nanocomposite suspensions until the MIC was determined.

\section{Effect of Strep-CS-MNPs on microbial growth}

In order to quantitatively evaluate the effect of the assynthesized Strep-CS-MNPs on the growth kinetics of different microorganisms, the plate colony-counting method was employed as described previously. ${ }^{18}$ Prior to treatment with the Strep-CS-MNPs, the bacterial cultures of Staphylococcus aureus (ATCC ${ }^{\circledR} 43300^{\mathrm{TM}}$ ), Pseudomonas aeruginosa $\left(\mathrm{ATCC}^{\circledR} 27853^{\mathrm{TM}}\right)$, Escherichia coli $\left(\mathrm{ATCC}^{\circledR}\right.$ $25922^{\mathrm{TM}}$ ), and Candida albicans $\left(\mathrm{ATCC}^{\circledR} 20408^{\mathrm{TM}}\right.$ ) were obtained from the ATCC and were hydrated and streaked for isolation on tryptic soy agar plates. Following growth, a single isolated colony was selected and used to inoculate 3 $\mathrm{mL}$ of $20 \%$ tryptic soy broth (TSB) medium. The bacterial culture was grown on a shaking incubator set at $200 \mathrm{rpm}$ for 18 hours at $37^{\circ} \mathrm{C}$. The resulting bacterial suspension was then adjusted to have an optical density (OD) $)_{570}$ of 0.52 , corresponding to a bacterial density of $10^{9}$ colony-forming unit (CFU)/mL. Then, the bacterial suspension was serially diluted over a 4-log range to a bacterial density of $10^{4} \mathrm{CFU} /$ $\mathrm{mL}$ using $20 \%$ TSB. A volume of $1 \mathrm{~mL}$ of the bacterial suspension was treated with the nanocomposite suspension at concentrations of 10 and $20 \mathrm{mg} / \mathrm{mL}$, respectively, in separate wells of a 24-well plate, and allowed to incubate for 1 hour at $37^{\circ} \mathrm{C}$. The number of CFUs after treatment was determined using the plate-counting method, ${ }^{18}$ following plating on tryptic soy agar plates. The experiment was repeated in triplicate.

The percentage of inhibition of the nanocomposites against each microorganism was calculated, as described previously ${ }^{18}$ and according to the following equation:

$$
\text { Inhibition rate }=1-\left[\mathrm{CFU}_{\text {treated }} / \mathrm{CFU}_{\text {control }}\right] \times 100
$$

The efficiency of the nanoparticles in inhibiting the growth of microorganisms was determined by differences in the equivalent number of CFUs before and after treatment as the percentage of microbes that were inhibited by the particles, and inhibition rate was calculated by using Equation 1.

\section{Determination of nanocomposite MICs}

The MICs of the nanocomposite against different microorganisms were determined using the broth microdilution method, according to Clinical and Laboratory Standards Institute (CLSI) guidelines. The MICs were determined over a range of $10-2,000 \mu \mathrm{g} / \mathrm{mL}$ by a two fold serial dilution method.

\section{Statistical analysis}

Unpaired $t$-tests were used to compare the MICs of Strep nanocomposites against $M$. tuberculosis. Statistical analysis was used to compare the percentage inhibition of Strep nanocomposites against different microorganisms using two-way analysis of variance (ANOVA) tests. The Prism V6.01 statistical software (GraphPad, San Diego, CA, USA) was used for data management and statistical analysis. ANOVA followed by Student's $t$-tests were used to determine the differences between the mean cell viabilities ( $\%)$. All data are shown as the mean \pm standard deviation, unless indicated differently.

\section{Results and discussion XRD analysis}

Figure 1A-C shows the XRD patterns of bare (unloaded), CS-MNPs, and Strep-CS-MNPs, respectively. The six characteristic peaks at $2 \theta=30,35,43,53,57$ and $62^{\circ}$ represent the Miller Indices of 220, 311, 400, 422, 511, and 440 planes. This result indicates that the MNPs did not contain hematite $\left(\alpha-\mathrm{Fe}_{2} \mathrm{O}_{3}\right)$, goethite, or other iron hydroxides. The lattice constant a was estimated for MNPs and CS-MNPs given at 8.395 and $8.392 \AA$, respectively. These values are close to that of magnetite (8.396 $\AA$ ), indicating that both samples were mainly magnetite and not maghemite, which has a value of $8.352 \AA .^{19}$ 


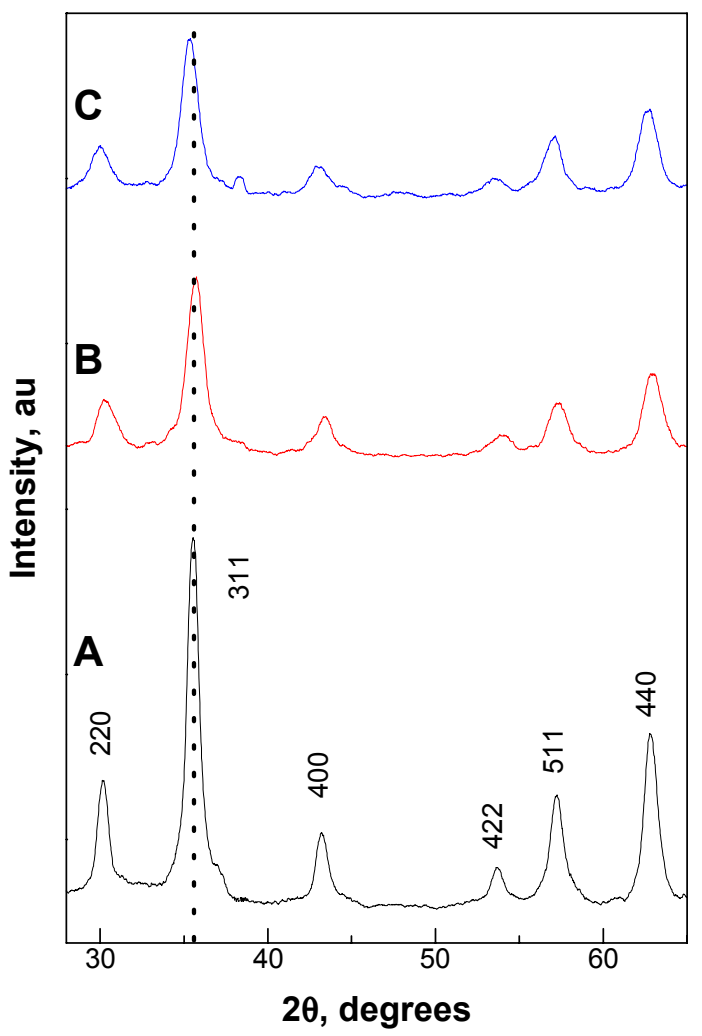

Figure I Powder X-ray diffraction patterns of the MNPs (black line A), CS-MNPs (red line B), and Strep-CS-MNPs nanocomposites (blue line $\mathbf{C}$ ).

Abbreviations: MNPs, magnetic nanoparticles; CS-MNPs, chitosan-coated magnetic nanoparticles; Strep-CS-MNPs, streptomycin-loaded, chitosan-coated magnetic nanoparticles.

The MIC of the Strep-CS-MNP nanocomposite against M. tuberculosis was $732 \mu \mathrm{g} / \mathrm{mL}$. This provides strong evidence of the anti-TB activity of the present nanocomposite. As shown in Figures 2B and 3, the nanocomposite was found to be more active against Gram-negative bacteria, specifically $P$. aeruginosa (MIC $=0.005 \mu \mathrm{g} / \mathrm{mL}$ ) and E. coli (MIC $=9.8 \mu \mathrm{g} / \mathrm{mL}$ ), as compared to Gram-positive bacteria S. aureus ( $\mathrm{MIC}=10 \mu \mathrm{g} / \mathrm{mL}$ ).

Here, we have synthesized MNPs coated with CS (CSMNPs) and the last synthesis was loaded with Strep to create Strep-CS-MNPs. From XRD analysis, we can see that MNPs, CS-MNPs, and Strep-CS-MNP nanocomposites contained mainly magnetite $\mathrm{Fe}_{3} \mathrm{O}_{4}$. The antimicrobial analysis of the Strep-CS-MNPs showed that the nanoparticles possessed antibacterial activity against a wide range of microorganisms, including TB. The antimicrobial and anti-TB activities of the developed Strep nanocomposites is considered a prototype for the development of other nano-antibiotics, based on other nano-polymers which will find a wide range of biomedical applications. Importantly, MNPs, such as those formulated here, can be directed to discrete locations in the body, where microorganisms may be, and can be detected via MRI to

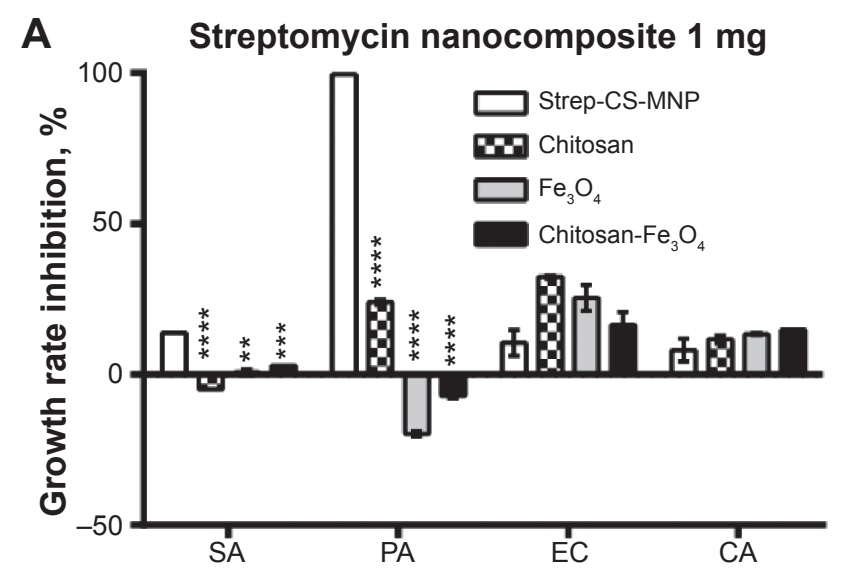

\section{B Streptomycin nanocomposite $2 \mathrm{mg}$}

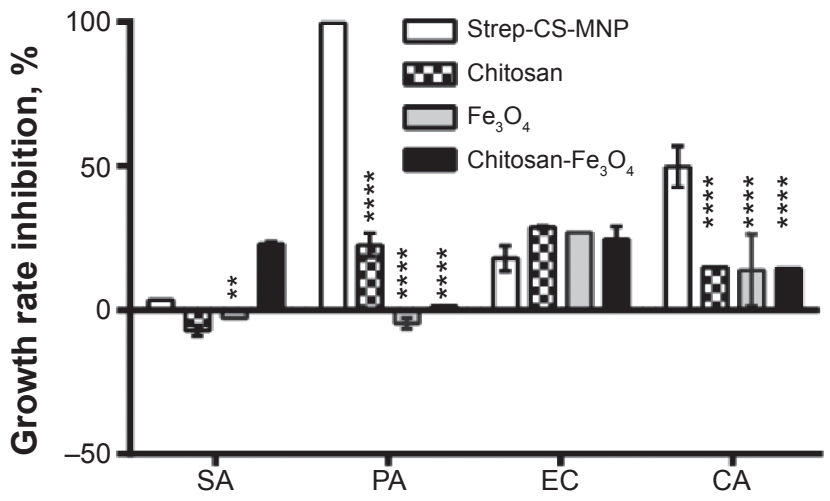

Figure 2 Effect of streptomycin-loaded, chitosan-coated magnetic nanoparticles (Strep-CS-MNPs) on the inhibition of microbial growth using the plate colonycounting method at two concentrations: (A) I mg and (B) $2 \mathrm{mg}$.

Notes: The data display the mean of the MIC \pm SEM. Each time point represents the mean growth rate inhibition \pm SEM. Asterisks indicate growth inhibition values of components that are significantly different from the growth rate inhibition values of Strep-CS-MNPs derived by using statistical analysis of two-way ANOVA, as follows: $* * * * P<0.0001$; *** $P<0.001 ; * * P<0.01$.

Abbreviations: CFU, colony-forming unit; SA, Staphylococcus aureus; PA, Pseudomonas aeruginosa; EC, Escherichia coli; CA, Candida albicans; MIC, minimum inhibitory concentration; SEM, standard error of the mean; ANOVA, analysis of variance.

indicate microorganism location. As such, the MNPs formulated here are strong candidates for detecting microorganism presence and killing such microorganisms at the same time, this dual purpose represents a new theranostic approach for numerous anti-microbial and anti-TB applications. Future studies should include in vivo analysis and should also determine the stability of these novel MNPs. In this context, the use of different antimicrobial nanoparticles is a promising field for overcoming the reduced effectiveness of currently used antimicrobial agents, which is due to the increasing resistance of bacteria.

\section{Conclusion}

This study introduces for the first time a new type of MNP with microorganism theranostic properties as a potential tool 


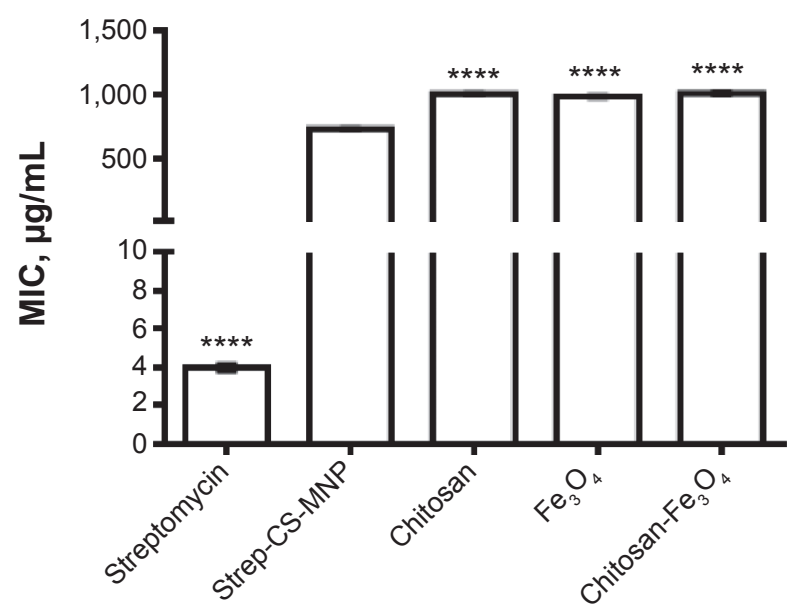

Figure 3 Minimum inhibitory concentrations (MICs; $\mu \mathrm{g} / \mathrm{mL}$ ) of streptomycinloaded, chitosan-coated magnetic nanoparticles (Strep-CS-MNPs), as compared to components against Mycobacterium tuberculosis, as determined by the Mycobacteria Growth Indicator Tube (MGIT TM) with BACTECTM MGIT 960 growth supplement for drug susceptibility testing (DST), and measured by the MGIT 960 instrument (BD, Franklin Lakes, NJ, USA).

Notes: The data display the mean of the MIC \pm SEM. The statistical significance was calculated using a one-way ANOVA for unpaired values between Strep-CS-MNPs and components, with the significance level indicated by asterisks (**** $P<0.000 \mathrm{I}$ ). Abbreviations: SEM, standard error of the mean; ANOVA, analysis of variance.

to both diagnose and treat diverse microbial and tuberculosis infections. Specifically, MNPs were synthesized by the coprecipitation of $\mathrm{Fe}^{2+}$ and $\mathrm{Fe}^{3+}$ iron salts in alkali media. MNPs were coated by CS to produce CS-MNPs. Strep was loaded onto the surface of CS-MNPs to form a Strep-CSMNP nanocomposite. For the first time, it was found that the nanoparticles had antimicrobial activities against the tested microorganisms (albeit with a more pronounced effect against Gram-negative than Gram-positive bacteria), and thus, should be further studied as a novel nano-antibiotic for numerous antimicrobial and antituberculosis applications. Moreover, since these nanoparticle bacteria fighters are magnetic, one can easily envision magnetic field direction of these nanoparticles to fight unwanted microorganism presence on demand.

\section{Acknowledgments}

The authors would like to thank the Ministry of Higher Education of Malaysia for grant number GP-IPB/2013/9425800, used for the preparation of the Strep nanocomposite. The authors would also like to thank Northeastern University, Boston, MA, USA, for funding the anti-TB experiments.

\section{Disclosure}

The authors report no conflicts of interest in this work.

\section{References}

1. Andrade AL, Souza DM, Pereira MC, Fabris JD, Domingues RZ. pH effect on the synthesis of magnetite nanoparticles by the chemical reduction-precipitation method. Quimica Nova. 2010;33(3):524-527.

2. Edelstein AS, Cammaratra RC. Nanomaterials: Synthesis, Properties and Applications. Boca Raton, FL: CRC Press; 1998.

3. Bumb A, Brechbiel MW, Choyke PL, et al. Synthesis and characterization of ultra-small superparamagnetic iron oxide nanoparticles thinly coated with silica. Nanotechnology. 2008;19(33):335601.

4. Bonnemain B. Superparamagnetic agents in magnetic resonance imaging: physicochemical characteristics and clinical applications a review. J Drug Target. 1998;6(3):167-174.

5. Babes L, Denizot B, Tanguy G, Le Jeune JJ, Jallet P. Synthesis of iron oxide nanoparticles used as MRI contrast agents: a parametric study. J Colloid Interface Sci. 1999;212(2):474-482.

6. Prucek R, Tuček J, Kilianová M, et al. The targeted antibacterial and antifungal properties of magnetic nanocomposite of iron oxide and silver nanoparticles. Biomaterials. 2011;32(21):4704-4713.

7. Chambers HF, Deleo FR. Waves of resistance: Staphylococcus aureus in the antibiotic era. Nat Rev Microbiol. 2009;7(9):629-641.

8. Falagas ME, Bliziotis IA. Pandrug-resistant Gram-negative bacteria: the dawn of the post-antibiotic era? Int J Antimicrob Agents 2007;29(6):630-636.

9. Spellberg B, Guidos R, Gilbert D, et al; Infectious Diseases Society of America. The epidemic of antibiotic-resistant infections: a call to action for the medical community from the Infectious Diseases Society of America. Clin Infect Dis. 2008;46(2):155-164.

10. Levy SB, Marshall B. Antibacterial resistance worldwide: causes, challenges and responses. Nat Med. 2004;10(12 Suppl):S122-S129.

11. Huh AJ, Kwon YJ. "Nanoantibiotics": a new paradigm for treating infectious diseases using nanomaterials in the antibiotics resistant era. $J$ Control Release. 2011;156(2):128-145.

12. Sharma A, Kumar Arya D, Dua M, Chhatwal GS, Johri AK. Nanotechnology for targeted drug delivery to combat antibiotic resistance. Expert Opin Drug Deliv. 2012;9(11):1325-1332.

13. Antimicrobial resistance: global report on surveillance 2014. World Health Organization (WHO); 2014. Available from: http://www.who. int/drugresistance/documents/surveillancereport/en/. Accessed September 17, 2014.

14. Hussein-Al-Ali SH, El Zowalaty ME, Hussein MZ, Ismail M, Webster TJ. Synthesis, characterization, controlled release, and antibacterial studies of a novel streptomycin chitosan magnetic nanoantibiotic. Int $J$ Nanomedicine. 2014;9:549-557.

15. Zhu Y, Wu Q. Synthesis of magnetite nanoparticles by precipitation with forced mixing. J Nanoparticle Res. 1999;1(3):393-396.

16. Rüsch-Gerdes S, Pfyffer GE, Casal M, Chadwick M, Siddiqi S. Multicenter laboratory validation of the BACTEC MGIT 960 technique for testing susceptibilities of Mycobacterium tuberculosis to classical second-line drugs and newer antimicrobials. J Clin Microbiol. 2006;44(3):688-692.

17. Walters SB, Hanna BA. Testing of susceptibility of Mycobacterium tuberculosis to isoniazid and rifampin by mycobacterium growth indicator tube method. J Clin Microbiol. 1996;34(6):1565-1567.

18. Usman MS, El Zowalaty ME, Shameli K, Zainuddin N, Salama M, Ibrahim NA. Synthesis, characterization, and antimicrobial properties of copper nanoparticles. Int J Nanomedicine. 2013;8:4467-4479.

19. Shan Z, Yang WS, Zhang X, Huang QM, Ye H. Preparation and characterization of carboxyl-group functionalized superparamagnetic nanoparticles and the potential for bio-applications. J Braz Chem Soc. 2007;18(7):1329-1335. 


\section{Publish your work in this journal}

The International Journal of Nanomedicine is an international, peerreviewed journal focusing on the application of nanotechnology in diagnostics, therapeutics, and drug delivery systems throughout the biomedical field. This journal is indexed on PubMed Central, MedLine, CAS, SciSearch $\AA$, Current Contents $\AA /$ Clinical Medicine,
Journal Citation Reports/Science Edition, EMBase, Scopus and the Elsevier Bibliographic databases. The manuscript management system is completely online and includes a very quick and fair peer-review system, which is all easy to use. Visit http://www.dovepress.com/ testimonials.php to read real quotes from published authors.

Submit your manuscript here: http://www.dovepress.com/international-journal-of-nanomedicine-journal 